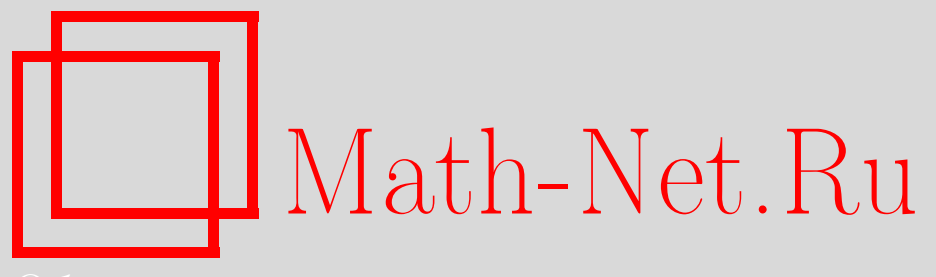

А. Н. Ширяев, М. Йор, К вопросу о стохастических интегральных представлениях функционалов от броуновского движения. I, Теория вероятн. и ее примен., 2003, том 48, выпуск 2, 375-385

DOI: https://doi.org/10.4213/tvp290

Использование Общероссийского математического портала Math-Net.Ru подразумевает, что вы прочитали и согласны с пользовательским соглашением

http://www . mathnet.ru/rus/agreement

Параметры загрузки:

IP: 3.82 .47 .9

26 апреля 2023 г., 15:49:59

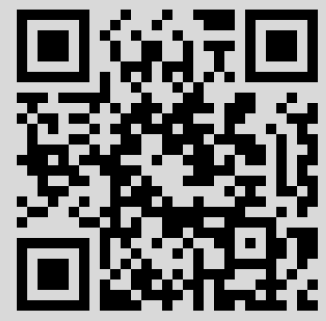




\section{К ВОПРОСУ О СТОХАСТИЧЕСКИХ ИНТЕГРАЛЬНЫХ ПРЕДСТАВЛЕНИЯХ ФУНКЦИОНАЛОВ ОТ БРОУНОВСКОГО ДВИЖЕНИЯ. ${ }^{1)}$}

Для функционалов $S=S(\omega)$ от броуновского движения предлагается метод отыскания стохастических интегральных представлений, основанный на использовании формулы Ито для ассоциированного с $S$ мартингала Леви. В качестве иллюстрации метода рассматриваются функционалы «максимального» типа: $S_{T}, S_{T_{-a}}, S_{g_{T}}$ и $S_{\theta_{T}}$, где $S_{T}=\max _{t \leqslant T} B_{t}, S_{T_{-a}}=\max _{t \leqslant T_{-a}} B_{t}$ с $T_{-a}=\inf \{t>0$ : $\left.B_{t}=-a\right\}, a>0$, и $S_{g_{T}}=\max _{t \leqslant g_{T}} B_{t}, S_{\theta_{T}}=\max _{t \leqslant \theta_{T}} B_{t}, g_{T}$ и $\theta_{T}$ - немарковские моменты: $g_{T}-$ момент последнего нуля броуновского движения на $[0, T]$, а $\theta_{T}-$ момент, когда броуновское движение достигает на $[0, T]$ своего максимального значения.

Ключевые слова и фразы: броуновское движение, марковские моменты, немарковские моменты, стохастический интеграл, формула Ито.

\section{§1. Введение}

1.1. Пусть $B=\left(B_{t}\right)_{t \geqslant 0}-$ стандартное броуновское движение, заданное на полном вероятностном пространстве $(\Omega, \mathscr{F}, \mathbf{P})$. Будем обозначать $\mathbb{F}=\left(\mathscr{F}_{t}\right)_{t \geqslant 0}$ фильтрацию, порожденную броуновским движением $\left(\mathscr{F}_{t}=\sigma\left(B_{s}, s \leqslant t\right), t \geqslant 0\right.$, где все рассматриваемые $\sigma$-алгебры предполагаются пополненными множествами из $\mathscr{F}$ нулевой Р-вероятности; полагаем также $\left.\mathscr{F}_{\infty}=\sigma\left(\bigcup_{t \geqslant 0} \mathscr{F}_{t}\right)\right)$.

Хорошо известно (см. [1]-[3]), что если $S=S(\omega)$ есть $\mathscr{F}_{\infty}$-измеримая случайная величина (т.е. $\mathscr{F}_{\infty}$-измеримый функционал от броуновского движения) с $\mathbf{E} S^{2}<\infty\left(S \in L^{2}\left(\Omega, \mathscr{F}_{\infty}, \mathbf{P}\right)\right)$, то найдется такой процесс $Y=\left(Y_{t}\right)_{t \geqslant 0}$, согласованный с фильтрацией $\mathbb{F}$ (т.е. $Y_{t}-\mathscr{F}_{t}$-измеримы,

* Математический институт им. В. А. Стеклова РАН, ул. Губкина, 8, 119991 Москва, ГСП-1, Россия; e-mail: shiryaev@mi.ras.ru

** Laboratoire de Probabilités et Modèles Aléatoires, Université Pierre et Marie Curie, Boîte courrier 188, 4 place Jussieu, 75252 Paris Cedex 05, France.

1) Работа выполнена при поддержке РФФИ, грант 02-01-00834, и Франко-русского центра им. А. М. Ляпунова (МГУ-INRIA), проект 3-97. 
$t \geqslant 0)$, что имеет место следующее стохастическое интегральное представление:

$$
S=\mathbf{E} S+\int_{0}^{\infty} Y_{t} d B_{t}
$$

где

$$
\mathbf{E} \int_{0}^{\infty} Y_{t}^{2} d t<\infty
$$

Следует подчеркнуть, что хотя формула (1) принципиально и решает вопрос о представлении $S$ в виде стохастического интеграла, отыскание явных выражений для $Y_{t}, t \geqslant 0$, является делом довольно-таки непростым.

Известен один достаточно общий результат, называемый «формулой Кларка-Окона», который дает для $Y_{t}$ следующее выражение:

$$
Y_{t}=\mathbf{E}\left(D S(t, \infty] \mid \mathscr{F}_{t}\right),
$$

где $D S$ - производная Маллявена (см., например, [4]).

Однако даже в сравнительно простых случаях вычисление $Y_{t}$ по формуле (3) требует значительных усилий.

1.2. Подход к нахождению явных формул для $Y_{t}, t \geqslant 0$, применяемый в настоящей работе, иной и состоит в идейном отношении в следующем.

Свяжем с функционалом $S$ ассочиированный мартингал Леви

$$
\mathbf{M}_{t}=\mathbf{E}\left(S \mid \mathscr{F}_{t}\right), \quad t \geqslant 0,
$$

и попытаемся представить $\mathbf{E}\left(S \mid \mathscr{F}_{t}\right)(\omega)$ в виде $F\left(t, \omega ; B_{t}(\omega)\right)$, где последний функционал таков, что к нему возможно применение (обобщенной) формуль Ито. (См., например, [8], [9]; ср. также с соответствуюшими методами в теории оптимальной нелинейной фильтрации, [5].)

По самой своей структуре формула Ито содержит стохастический интеграл по броуновскому движению, который и должен дать (с точностью до константы) стохастическое интегральное представление для мартингала $\left(\mathbf{M}_{t}\right)_{t \geqslant 0}$. Поскольку по теореме Леви

$$
\left.\mathbf{M}_{t}=\mathbf{E}\left(S \mid \mathscr{F}_{t}\right) \longrightarrow \mathbf{E}\left(S \mid \mathscr{F}_{\infty}\right)=S \quad \text { (P-п.н. }\right),
$$

то, тем самым, получаем, как следствие, и требуемое стохастическое интегральное представление для функционала $S$.

1.3. Осуществление намеченной программы будет ниже продемонстрировано на примерах нескольких функционалов $\lambda$ «максимального» типа. Именно, пусть

$$
S_{t}=\max _{u \leqslant t} B_{u} .
$$

В первой части работы в $\S 2$ рассматривается стохастическое интегральное представление для функционала $S=S_{T}$, где $T$ - некоторая константа, т.е. для случая $S=\max _{u \leqslant T} B_{u}$. 
В $\S 3$ исследуется случай $S=S_{T_{-a}}$, т.е. $S=\max _{u \leqslant T_{-a}} B_{u}$, где

$$
T_{-a}=\inf \left\{t>0: B_{t}=-a\right\}, \quad a>0 .
$$

Моменты $T$ и $T_{-a}$ являются, очевидно, марковскими моментами. Во второй части работы рассматриваются вопросы интегрального представления для функционалов

$$
S=S_{g_{T}} \quad \text { и } \quad S=S_{\theta_{T}},
$$

где $g_{T}$ и $\theta_{T}$ - немарковские моменты:

$g_{T}$ - момент последнего нуля броуновского движения на $[0, T]$ с $g_{T}=T$, если такого момента нет; и

$\theta_{T}$ - момент, когда броуновское движение достигает на $[0, T]$ своего максимального значения.

Эти два случая рассматриваются соответственно в $\S 4$ и 5 второй части работы.

В заключение настоящего введения отметим, что, помимо собственно результатов относительно стохастических интегральных представлений для «частичных» максимумов $S_{T}, S_{T_{-a}}, S_{g_{T}}$ и $S_{\theta_{T}}$, данная работа содержит также ряд нетрадиционных методов и приемов стохастического анализа (см., например, лемму 1 о стохастическом интегральном соотношении для индикатора $I\left(T_{a}<T\right)$ марковского момента $\left.T_{a}, a>0\right)$, которые, как нам представляется, могут быть полезными и в других задачах теории случайных процессов.

\section{§ 2. Случай $S_{T}=\max _{t \leqslant T} B_{t}$}

2.1. Вопрос о стохастическом интегральном представлении функционала $S_{T}$ рассматривался в ряде работ (см., например, [1], [2], [4], [6]), согласно которым справедливо следующее утверждение.

Теорема 1. Для $S_{T}$ имеет место стохастическое интегральное представление:

$$
S_{T}=\mathbf{E} S_{T}+2 \int_{0}^{T}\left[1-\Phi\left(\frac{S_{t}-B_{t}}{\sqrt{T-t}}\right)\right] d B_{t}
$$

где $S_{t}=\max _{u \leqslant t} B_{u}$

$$
\mathbf{E} S_{T}=\mathbf{E} \max _{t \leqslant T} B_{t}=\mathbf{E}\left|B_{T}\right|=\sqrt{\frac{2 T}{\pi}}, \quad \Phi(x)=\frac{1}{\sqrt{2 \pi}} \int_{-\infty}^{x} e^{-y^{2} / 2} d y .
$$

Ниже будут приведены два способа доказательства представления (4), основанные на изложенной выше стратегии, опирающейся на применение формулы Ито к ассоциированному мартингалу $\left(\mathbf{E}\left[S \mid \mathscr{F}_{t}\right]\right)_{t \geqslant 0}$. 
Д о к а з а т е л ь с т в о. Первый способ. Для каждой неотрицательной случайной величины $\xi$ справедливо представление

$$
\xi=\int_{0}^{\infty} I(a<\xi) d a .
$$

Следовательно,

$$
S_{T}=\max _{t \leqslant T} B_{t}=\int_{0}^{\infty} I\left(a<\max _{t \leqslant T} B_{t}\right) d a=\int_{0}^{\infty} I\left(T_{a}<T\right) d a,
$$

где

$$
T_{a}=\inf \left\{t \geqslant 0: B_{t}=a\right\}, \quad a \geqslant 0 .
$$

Следующий результат, интересный и сам по себе, дает стохастическое интегральное соотношение для момента остановки $T_{a}$.

Лемма 1. Для всякого $a>0$ (P-n.н.)

$$
I\left(T_{a}<T\right)=\mathbf{P}\left\{T_{a}<T\right\}+2 \int_{0}^{T_{a} \wedge T} \varphi_{T-t}\left(B_{t}-a\right) d B_{t},
$$

əde

$$
\varphi_{t}(a)=\frac{1}{\sqrt{2 \pi t}} e^{-a^{2} /(2 t)}
$$

Д о к а з а т е л ь с т в о. Рассмотрим стохастическую экспоненту

$$
\mathscr{E}_{t}(\lambda)=e^{\lambda B_{t}-\lambda^{2} t / 2}, \quad \lambda \in \mathbf{R} .
$$

По теореме Дуба об остановке для мартингалов находим, что

$$
\mathbf{E}\left(\mathscr{E}_{T_{a}}(\lambda) \mid \mathscr{F}_{t}\right)=\mathscr{E}_{T_{a} \wedge t}(\lambda) \quad \text { (P-П.н.). }
$$

По формуле Ито

$$
d \mathscr{E}_{t}(\lambda)=\lambda \mathscr{E}_{t}(\lambda) d B_{t}, \quad \mathscr{E}_{0}(\lambda)=1 .
$$

Отсюда и из (9)

$$
\mathbf{E}\left(\mathscr{E}_{T_{a}}(\lambda) \mid \mathscr{F}_{t}\right)=1+\lambda \int_{0}^{T_{a} \wedge t} e^{\lambda B_{s}-\lambda^{2} s / 2} d B_{s},
$$

и, поскольку $\mathscr{E}_{T_{a}}(\lambda)=e^{\lambda a-\lambda^{2} T_{a} / 2}$ (Р-п.н.),

$$
\mathbf{E}\left(e^{-\lambda^{2} T_{a} / 2} \mid \mathscr{F}_{t}\right)=e^{-\lambda a}+\lambda \int_{0}^{T_{a} \wedge t} e^{-\lambda\left(a-B_{\bullet}\right)-\lambda^{2} s / 2} d B_{s} .
$$

В частности, полагая $t \rightarrow \infty$, получаем, что (Р-п.н.)

$$
e^{-\lambda^{2} T_{a} / 2}=e^{-\lambda a}+\lambda \int_{0}^{T_{a}} e^{-\lambda\left(a-B_{s}\right)-\lambda^{2} s / 2} d B_{s} .
$$

Преобразуем правые части в (12), (13). С этой целью положим

$$
\gamma_{a}(t)=\frac{d}{d t} \mathbf{P}\left\{T_{a} \leqslant t\right\}
$$


Известно (см. [3], [7]), что

$$
\gamma_{a}(t)=\frac{a}{\sqrt{2 \pi t^{3}}} e^{-a^{2} /(2 t)} \quad\left(=-\frac{\partial}{\partial a} \varphi_{t}(a)\right)
$$

Из (9)

$$
\mathbf{E} e^{\lambda^{2} T_{a} / 2}=e^{-\lambda a}
$$

Поэтому для всякого $c>0$

$$
\begin{aligned}
\lambda e^{-\lambda c} & =-\frac{d}{d c} e^{-\lambda c}=-\frac{d}{d c}\left(\mathbf{E} e^{-\lambda^{2} T_{c} / 2}\right) \\
& =-\frac{d}{d c} \int_{0}^{\infty} e^{-\lambda^{2} t / 2} \gamma_{c}(t) d t=-\int_{0}^{\infty} e^{-\lambda^{2} t / 2}\left(\frac{\partial}{\partial c} \gamma_{c}(t)\right) d t \\
& =\int_{0}^{\infty} e^{-\lambda^{2} t / 2}\left(\frac{\partial^{2}}{\partial c^{2}} \varphi_{t}(c)\right) d t=2 \int_{0}^{\infty} e^{-\lambda^{2} t / 2}\left(\frac{\partial}{\partial t} \varphi_{t}(c)\right) d t
\end{aligned}
$$

поскольку

$$
\frac{\partial \varphi_{t}(c)}{\partial t}=\frac{1}{2} \frac{\partial^{2} \varphi_{t}(c)}{\partial c^{2}}
$$

Из (12), (16) и (17), учитывая, что $B_{s}<a$ на множестве $\left\{\omega: s<T_{a}\right\}$, находим

$$
\begin{aligned}
& \mathbf{E}\left(e^{-\lambda^{2} T_{a} / 2} \mid \mathscr{F}_{t}\right) \\
& \quad=\mathbf{E} e^{-\lambda^{2} T_{a} / 2}+2 \int_{0}^{T_{a} \wedge t}\left[\int_{0}^{\infty} e^{-\lambda^{2} u / 2}\left(\frac{\partial}{\partial u} \varphi_{u}\left(a-B_{s}\right)\right) d u\right] e^{-\lambda^{2} s / 2} d B_{s} \\
& \quad=\mathbf{E} e^{-\lambda^{2} T_{a} / 2}+2 \int_{0}^{T_{a} \wedge t}\left[\int_{0}^{\infty} e^{-\lambda^{2}(u+s) / 2}\left(\frac{\partial}{\partial u} \varphi_{u}\left(B_{s}-a\right)\right) d u\right] d B_{s} .
\end{aligned}
$$

Отсюда для достаточно «хороших» и не слишком быстро растущих функций $f=f(x)$ получаем (детали см. во второй части работы), что (P-п.н.)

$\mathbf{E}\left(f\left(T_{a}\right) \mid \mathscr{F}_{t}\right)=\mathbf{E} f\left(T_{a}\right)+2 \int_{0}^{T_{a} \wedge t}\left[\int_{0}^{\infty} f(u+s)\left(\frac{\partial}{\partial u} \varphi_{u}\left(B_{s}-a\right)\right) d u\right] d B_{s}$.

В частности, полагая $f(t)=I(t<T)$, приходим к следующему:

$$
\begin{aligned}
I\left(T_{a}<T\right)= & \mathbf{P}\left\{T_{a}<T\right\} \\
& +2 \int_{0}^{T_{a}}\left[\int_{0}^{\infty} I(u+s<T)\left(\frac{\partial}{\partial u} \varphi_{u}\left(B_{s}-a\right)\right) d u\right] d B_{s} \\
= & \mathbf{P}\left\{T_{a}<T\right\}+2 \int_{0}^{T_{a}}\left[\int_{0}^{T-s} \frac{\partial}{\partial u} \varphi_{u}\left(B_{s}-a\right) d u\right] d B_{s} \\
= & \mathbf{P}\left\{T_{a}<T\right\}+2 \int_{0}^{T_{a} \wedge t} \varphi_{T-s}\left(B_{s}-a\right) d B_{s},
\end{aligned}
$$

что и доказывает требуемое представление. Лемма 1 доказана. 
2.2. Из представления (6) и из (5) видим, что

$$
\begin{aligned}
S_{T}= & \max _{t \leqslant T} B_{t}=\int_{0}^{\infty} I\left(T_{a}<T\right) d a \\
= & \int_{0}^{\infty} \mathbf{P}\left\{T_{a}<T\right\} d a+2 \int_{0}^{\infty}\left[\int_{0}^{T_{a} \wedge T} \varphi_{T-s}\left(B_{s}-a\right) d B_{s}\right] d a \\
= & \int_{0}^{\infty} \mathbf{P}\left(\max _{s \leqslant T} B_{s}>a\right) d a \\
& +2 \int_{0}^{\infty} \int_{0}^{\infty} I\left(s<T_{a} \wedge T\right) \varphi_{T-s}\left(B_{s}-a\right) d B_{s} d a \\
= & \mathbf{E} \max _{s \leqslant T} B_{s}+2 \int_{0}^{T}\left[\int_{0}^{\infty} I\left(s<T_{a}\right) \varphi_{T-s}\left(B_{s}-s\right) d a\right] d B_{s} \\
= & \mathbf{E} S_{T}+2 \int_{0}^{T}\left[\int_{0}^{\infty} \varphi_{T-u}\left(B_{u}-a\right) I\left(S_{u}<a\right) d a\right] d B_{u} .
\end{aligned}
$$

Здесь

$$
\begin{aligned}
\int_{0}^{\infty} & \varphi_{T-u}\left(B_{u}-a\right) I\left(S_{u}<a\right) d a=\int_{S_{u}}^{\infty} \varphi_{T-u}\left(B_{u}-a\right) d a \\
= & \int_{S_{u}}^{\infty} \frac{1}{\sqrt{2 \pi(T-u)}} \exp \left(-\frac{\left(B_{u}-a\right)^{2}}{2(T-u)}\right) d a \\
= & \frac{1}{\sqrt{2 \pi}} \int_{\left(S_{u}-B_{u}\right) / \sqrt{T-u}} e^{-a^{2} / 2} d a=1-\Phi\left(\frac{S_{u}-B_{u}}{\sqrt{T-u}}\right) .
\end{aligned}
$$

Из (22) и (23) получаем представление (4).

Следствие. Пусть $M_{T}=\min _{t \leqslant T} B_{t}$. Тогда

$$
M_{T}=\mathbf{E} M_{T}-2 \int_{0}^{T}\left[1-\Phi\left(\frac{B_{t}-M_{t}}{\sqrt{T-t}}\right)\right] d B_{t}
$$

Для доказательства достаточно лишь заметить, что если $M_{t}=$ $M_{t}(B)\left(=\min _{u \leqslant t} B_{u}\right)$ и $S_{t}=S_{t}(B)\left(=\max _{u \leqslant t} B_{u}\right)$, то $M_{t}(B)=-S_{t}(-B)$ и (24) следует из (4).

2.3. Доказ ат льст тво те оремы 1. Bmopoŭ cnocoб.

В приведенном выше доказательстве формулы (4) ключевыми являлись формула (5) и представление (6) для индикатора $I\left(T_{a}<T\right)$, интересное и само по себе.

Приводимый ниже способ отыскания представлений типа (4) состоит в непосредственном рассмотрении ассоциированных мартингалов с последующим применением формулы Ито. При этом рассматриваться будет не только интересующий нас процесс броуновского движения $B=\left(B_{t}\right)_{t \geqslant 0}$, но и более общие процессы, а именно процессы Леви.

Лемма 2. Пусть $X=\left(X_{t}\right)_{t \geqslant 0}-$ nроиесс Леви с $\mathbf{E} S_{T}<\infty$, где $T<\infty u S_{t}=\sup _{u \leqslant t} X_{u}$. Пycmb $F_{T-t}(s)=\mathbf{P}\left\{S_{T-t} \leqslant s\right\} u \mathscr{F}_{t}^{X}=$ 
$\sigma\left(X_{u}, u \leqslant t\right)$. Тогда для $t \leqslant T$

$$
\mathbf{E}\left(S_{T} \mid \mathscr{F}_{t}^{X}\right)=S_{t}+\int_{S_{t}-X_{t}}^{\infty}\left(1-F_{T-t}(u)\right) d u .
$$

Д о к а з ат е ль с т в о, в основном, следует п. 3 работы [6]. Из свойств процессов Леви следует, что

$$
\begin{aligned}
\mathbf{E}\left(S_{T} \mid \mathscr{F}_{t}^{X}\right) & =\mathbf{E}\left(\max \left(S_{t}, \sup _{t \leqslant u \leqslant T} S_{u}\right) \mid \mathscr{F}_{t}^{X}\right) \\
& =S_{t}+\mathbf{E}\left(\left(\sup _{t \leqslant u \leqslant T} S_{u}-S_{t}\right)^{+} \mid \mathscr{F}_{t}^{X}\right) \\
& =S_{t}+\mathbf{E}\left(\left[\sup _{t \leqslant u \leqslant T}\left(S_{u}-X_{t}\right)-\left(S_{t}-X_{t}\right)\right]^{+} \mid \mathscr{F}_{t}^{X}\right) \\
& =S_{t}+\left.\mathbf{E}\left(S_{T-t}-(s-x)\right)^{+}\right|_{x=X_{t}, s=S_{t}} .
\end{aligned}
$$

Воспользовавшись формулой $\mathbf{E}(\xi-c)^{+}=\int_{c}^{\infty} \mathbf{P}\{\xi>z\} d z$, из (26) находим требуемое представление (25).

Заметим, что правая часть в $(25)$ имеет вид $f\left(t, X_{t}, S_{t}\right)$, где

$$
f(t, x, s)=s+\int_{s-x}^{\infty}\left(1-F_{T-t}(u)\right) d u .
$$

В частности, если $X_{t}=B_{t}^{\mu}$, где $B_{t}^{\mu}=\mu t+B_{t}$ (броуновское движение со сносом), то применение формулы Ито к непрерывному мартингалу $f\left(t, B_{t}^{\mu}, S_{t}^{\mu}\right), t \leqslant T$, с $S_{t}^{\mu}=\max _{u \leqslant t} B_{u}^{\mu}$ дает следующее представление:

$$
\mathbf{E}\left(S_{T}^{\mu} \mid \mathscr{F}_{t}^{B}\right)=\mathbf{E} S_{T}^{\mu}+\int_{0}^{t} \frac{\partial f}{\partial x}\left(s, B_{s}^{\mu}, S_{s}^{\mu}\right) d B_{s}
$$

где $\partial f / \partial x$ есть частная производная функции $f=f(t, x, s)$.

Из (19) и (28) получаем

$$
\mathbf{E}\left(S_{T}^{\mu} \mid \mathscr{F}_{t}^{B}\right)=\mathbf{E} S_{T}^{\mu}+\int_{0}^{t}\left[1-F_{T-u}\left(S_{u}^{\mu}-B_{u}^{\mu}\right)\right] d B_{u} .
$$

В случае броуновского движения со сносом (см. [3], [7])

$$
F_{T-u}(s)=\mathbf{P}\left\{S_{T-u} \leqslant s\right\}=\Phi\left(\frac{s-\mu(T-u)}{\sqrt{T-u}}\right)-e^{2 \mu s} \Phi\left(\frac{-s-\mu(T-u)}{\sqrt{T-u}}\right) .
$$

Тем самым,

$$
\mathbf{E}\left(S_{T}^{\mu} \mid \mathscr{F}_{t}^{B}\right)=\mathbf{E} S_{T}^{\mu}+\int_{0}^{t} H_{u}^{\mu} d B_{u}
$$

где

$$
\begin{aligned}
H_{u}^{\mu}= & 1-\Phi\left(\frac{\left(S_{u}^{\mu}-B_{u}^{\mu}\right)-\mu(T-u)}{\sqrt{T-u}}\right) \\
& +\exp \left\{2 \mu\left(S_{u}^{\mu}-B_{u}^{\mu}\right)\right\} \Phi\left(\frac{-\left(S_{u}^{\mu}-B_{u}^{\mu}\right)-\mu(T-u)}{\sqrt{T-u}}\right)
\end{aligned}
$$

Если $\mu=0$, то из (30) и (31) получаем представление (4). 


\section{§3. Случай $S_{T_{-a}}=\max _{t \leqslant T_{-a}} B_{t}, a>0$}

3.1. Поскольку $\mathbf{E} S_{T_{-a}}=\infty$, то непосредственное обращение к ассоциированному мартингалу становится невозможным. Поэтому рассмотрим некоторую достаточно «хорошую» неотрицательную функцию $f=f(x)$ такую, что $\mathbf{E} f\left(S_{T_{-a}}\right)<\infty$. (См. далее условия в теореме 2.)

Обозначим $F=f\left(S_{T_{-a}}\right)$, и пусть $\left(F_{t}\right)_{t \geqslant 0}$ является ассоциированным с $F$ мартингалом Леви, $F_{t}=\mathbf{E}\left(F \mid \mathscr{F}_{t}^{B}\right)$.

Зафиксируем сейчас некоторое $t>0$. Тогда на множестве $\left\{t<T_{-a}\right\}$

$$
F_{t}=\mathbf{E}\left(f\left(S_{T_{-a}}\right) \mid \mathscr{F}_{t}^{B}\right)=\mathbf{E}\left[f\left(\max \left(S_{t}, \sup _{t \leqslant u \leqslant T_{-a}} B_{u}\right)\right) \mid \mathscr{F}_{t}^{B}\right] .
$$

Если положить $\widehat{B}_{u}=B_{u+t}-B_{t}, u \geqslant 0$, и $\widehat{T}_{-b}=\inf \left\{u>0: \widehat{B}_{u}=-b\right\}$, $b>0$, то найдем, что тождественно

$$
\begin{aligned}
\sup _{t \leqslant u \leqslant T_{-a}} B_{u} & =B_{t}+\sup _{t \leqslant u \leqslant T_{-a}}\left(B_{u}-B_{t}\right)=B_{t}+\sup _{0 \leqslant v \leqslant T_{-a}+t}\left(B_{v+t}-B_{t}\right) \\
& =B_{t}+\sup _{0 \leqslant u \leqslant \widehat{T}_{-\left(a+B_{t}\right)}}\left(B_{u+t}-B_{t}\right),
\end{aligned}
$$

где последнее равенство следует из того, что на множестве $\left\{t<T_{-a}\right\}$

$$
\begin{aligned}
\widehat{T}_{-\left(a+B_{t}\right)} & =\inf \left\{u \geqslant 0: \widehat{B}_{u}=-\left(a+B_{t}\right)\right\} \\
& =\inf \left\{u \geqslant 0: B_{u+t}-B_{t}=-\left(a+B_{t}\right)\right\} \\
& =\inf \left\{u \geqslant 0: B_{u+t}=-a\right\}=T_{-a}-t .
\end{aligned}
$$

Из (32) следует, что на $\left\{t<T_{-a}\right\}$

$$
\sup _{t \leqslant u \leqslant T_{-a}} B_{u}=B_{t}+\widehat{S}_{\widehat{T}_{-\left(a+B_{t}\right)}},
$$

где $\widehat{S}_{t}=\sup _{u \leqslant t} \widehat{B}_{u}$, и, значит, на этом множестве

$$
F_{t}=\mathbf{E}\left[f\left(\max \left(S_{t}, B_{t}+\widehat{S}_{\widehat{T}_{-\left(a+B_{t}\right.}}\right)\right) \mid \mathscr{F}_{t}\right] .
$$

3.2. Для дальнейшего упрощения правой части равенства (33) нам понадобится следующая (известная специалистам) лемма, приводимая вместе с доказательством для полноты изложения.

Лемма 3. Пусть $M=\left(M_{t}\right)_{t \geqslant 0}-$ непрерывный неотрииательный мартингал $\left(M \in \mathscr{M}^{\mathrm{c}}, M \geqslant 0\right)$ с $M_{0}=b>0$ и $M_{\infty} \equiv \lim _{t \rightarrow \infty} M_{t}=0$. Тогда

$$
\sup _{t \geqslant 0} M_{t} \stackrel{\text { law }}{=} \frac{b}{U}
$$

где $U=U[0,1]-$ случайная величина, равномерно распределенная на $[0,1]$, m.e.

$$
\mathbf{P}\left\{\sup _{t \geqslant 0} M_{t} \leqslant b+x\right\}=\frac{x}{b+x}, \quad x \geqslant 0 .
$$


Доказательст в о. Пусть $T_{b+x}=\inf \left\{t \geqslant 0: M_{t}=b+x\right\}$ и $\widetilde{M}_{t}=$ $M_{t \wedge T_{b+x}}$. Мартингал $\widetilde{M}=\left(\widetilde{M}_{t}\right)_{t \geqslant 0}$ является ограниченным и сверху, и снизу $\left(0 \leqslant \widetilde{M}_{t} \leqslant b+x\right)$. Поэтому согласно теореме Дуба об остановке $\mathbf{E} \widetilde{M}_{0}=\mathbf{E} \widetilde{M}_{T_{b+x}}$, и, значит,

$$
\begin{aligned}
b & =\mathbf{E} \widetilde{M}_{0}=\mathbf{E} \widetilde{M}_{T_{b+x}}=\mathbf{E} M_{T_{b+x}}=\mathbf{E} M_{T_{b+x}} I\left(T_{b+x}<\infty\right) \\
& =(b+x) \mathbf{P}\left\{T_{b+x}<\infty\right\}=(b+x) \mathbf{P}\left\{\sup _{t \geqslant 0} M_{t} \geqslant b+x\right\},
\end{aligned}
$$

что и доказывает равенство (34).

Применяя эту лемму к $M_{t}=b+\widehat{B}_{t \wedge \widehat{T}_{-b}}$, видим, что

$$
\mathbf{P}\left\{\sup _{t \leqslant \widehat{T}_{-b}} \widehat{B}_{t}>x\right\}=\mathbf{P}\left\{\sup _{t \geqslant 0} M_{t}>b+x\right\}=\frac{b}{b+x},
$$

или

$$
\mathbf{P}\left\{\sup _{t \leqslant \widehat{T}_{-b}} \widehat{B}_{t} \in d x\right\}=\frac{b d x}{(b+x)^{2}}, \quad x \geqslant 0 .
$$

Из (33) и (35), с учетом независимости броуновского движения $\widehat{B}=\left(\widehat{B}_{u}\right)_{u \geqslant 0}$ от $\mathscr{F}_{t}$, находим, что на множестве $\left\{t \leqslant T_{-a}\right\}$

$$
F_{t}=\int_{0}^{\infty} f\left(\max \left(S_{t}, B_{t}+x\right)\right) \frac{b d x}{(b+x)^{2}}, \quad b=a+B_{t} .
$$

Если $B_{t}+x \leqslant S_{t}$ (т.е. $x \leqslant S_{t}-B_{t}$ ), то

$$
\begin{aligned}
F_{t}= & \int_{0}^{S_{t}-B_{t}} f\left(S_{t}\right) \frac{b d x}{(b+x)^{2}}+\int_{S_{t}-B_{t}}^{\infty} f\left(B_{t}+x\right) \frac{b d x}{(b+x)^{2}} \\
= & f\left(S_{t}\right) \frac{S_{t}-B_{t}}{a+S_{t}}+\int_{0}^{\infty} f\left(S_{t}+y\right) \frac{b d y}{\left(a+S_{t}+y\right)^{2}} \\
= & f\left(S_{t}\right) \frac{S_{t}-B_{t}}{a+S_{t}}+\left(a+B_{t}\right) \int_{0}^{\infty} f\left(S_{t}+y\right) \frac{d y}{\left(a+S_{t}+y\right)^{2}} \\
= & {\left[f\left(S_{t}\right) \frac{S_{t}}{a+S_{t}}+a \int_{0}^{\infty} \frac{f\left(S_{t}+y\right)}{\left(a+S_{t}+y\right)^{2}} d y\right] } \\
& +B_{t}\left[-\frac{f\left(S_{t}\right)}{a+S_{t}}+\int_{0}^{\infty} \frac{f\left(S_{t}+y\right)}{\left(a+S_{t}+y\right)^{2}} d y\right] \equiv g\left(S_{t}\right)+B_{t} h\left(S_{t}\right),
\end{aligned}
$$

где

$$
\begin{aligned}
g(\sigma) & =\frac{\sigma f(\sigma)}{a+\sigma}+a \int_{0}^{\infty} \frac{f(\sigma+y)}{(a+\sigma+y)^{2}} d y, \\
h(\sigma) & =-\frac{f(\sigma)}{a+\sigma}+\int_{0}^{\infty} \frac{f(\sigma+y)}{(a+\sigma+y)^{2}} d y \\
& =\int_{0}^{\infty}[f(\sigma+y)-f(\sigma)] \frac{d y}{(a+\sigma+y)^{2}} .
\end{aligned}
$$


Процесс $S=\left(S_{t}\right)_{t \geqslant 0}$ является процессом ограниченной вариации. Поэтому из (37) по формуле Ито находим, что на множестве $\left\{t<T_{-a}\right\}$

$$
d F_{t}=\left[g^{\prime}\left(S_{t}\right)+B_{t} h^{\prime}\left(S_{t}\right)\right] d S_{t}+h\left(S_{t}\right) d B_{t} .
$$

Но $B_{t} h^{\prime}\left(S_{t}\right) d S_{t}=S_{t} h^{\prime}\left(S_{t}\right) d S_{t}$ (в смысле выполнения соответствующего интегрального соотношения). Отсюда и из (38), с учетом легко проверяемого равенства $g^{\prime}(\sigma)+\sigma h^{\prime}(\sigma)=0$, получаем, что на множестве $\left\{t \leqslant T_{-a}\right\}$

$$
F_{t}=F_{0}+\int_{0}^{t} h\left(S_{u}\right) d B_{u}=g(0)+\int_{0}^{t} h\left(S_{u}\right) d B_{u} .
$$

3 а м е ч а н и е. «Исчезновение» в (38) первого слагаемого в правой части можно было предвидеть, и не обращаясь к равенству $g^{\prime}(\sigma)+$ $\sigma h^{\prime}(\sigma)=0$, поскольку процесс $\left(F_{t}\right)_{t \geqslant 0}$ должен быть мартингалом, а стохастический дифференциал по $d B_{t}$ в интегральной форме дает локальный мартингал.

Из (39) предельным переходом $t \uparrow T_{-a}$ приходим к следующему утверждению.

Теорема 2. Пусть функиия $f=f(x), x \geqslant 0$, такова, что для $a>0, \sigma \geqslant 0$

$$
\int_{0}^{\infty} \frac{|f(\sigma+y)|}{(a+\sigma+y)^{2}} d y<\infty
$$

$u$

$$
\int_{0}^{T_{-a}} h^{2}\left(S_{u}\right) d u<\infty \quad(\mathbf{P}-\text { n.н. }) .
$$

Тогда справедливо следующее стохастическое интегральное представление:

$$
f\left(\max _{u \leqslant T_{-a}} B_{u}\right)=\mathbf{E} f\left(\max _{u \leqslant T_{-a}} B_{u}\right)+\int_{0}^{T_{-a}} h\left(S_{u}\right) d B_{u},
$$

əде

$$
\mathbf{E} f\left(\max _{u \leqslant T_{-a}} B_{u}\right)=\int_{0}^{\infty} \frac{a f(y)}{(a+y)^{2}} d y .
$$

Следствие. Из (40)

$$
I\left(\max _{u \leqslant T_{-a}} B_{u}>z\right)=\frac{a}{a+z}+\int_{0}^{T_{-a}} \frac{I\left(S_{u}<z\right)}{a+z} d B_{u} .
$$

Интересно отметить, что соотношение (42) равносильно тому, что

$$
(a+z) I\left(S_{T_{-a}}>z\right)=a+B_{T_{-a} \wedge T_{z}} .
$$

Это равенство на самом деле является очевидным. Действительно, на множестве $\left\{S_{T_{-a}}>z\right\}$ левая часть равна $a+z$, а правая часть также равна $a+z$, поскольку $B_{T_{-a} \wedge T_{z}}=z$ на множестве $\left\{S_{T_{-a}}>z\right\}$. Если же $S_{T_{-a}} \leqslant z$, то обе части в (43) равны нулю. 
3.3. Обратимся снова к представлению (42). Поскольку стохастический интеграл $\int_{0}^{T_{-a}} d B_{u}=-a$, то $(42)$ может быть переписано в виде

$$
I\left(\max _{u \leqslant T_{-a}} B_{u}>z\right)=-\int_{0}^{T_{-a}} \frac{I\left(z<S_{u}\right)}{a+z} d B_{u} .
$$

Отсюда

$$
\begin{aligned}
S_{T_{-a}} & =\max _{u \leqslant T_{-a}} B_{u}=\int_{0}^{\infty} I\left(S_{T_{-a}}>z\right) d z=-\int_{0}^{\infty}\left[\int_{0}^{T_{-a}} \frac{I\left(z<S_{u}\right)}{a+z} d B_{u}\right] d z \\
& =-\int_{0}^{T_{-a}}\left[\int_{0}^{\infty} \frac{I\left(z<S_{u}\right)}{a+z} d z\right] d B_{u}=-\int_{0}^{T_{-a}}\left[\int_{0}^{S_{u}} \frac{d z}{a+z}\right] d B_{u} \\
& =-\int_{0}^{T_{-a}} \ln \frac{a+S_{u}}{a} d B_{u} .
\end{aligned}
$$

Следовательно, справедливо стохастическое интегральное представление

$$
\max _{u \leqslant T_{-a}} B_{u}=-\int_{0}^{T_{-a}} \ln \left(1+\frac{S_{u}}{a}\right) d B_{u} .
$$

Как уже отмечалось, $\mathbf{E}_{\max _{u \leqslant T_{-a}}} B_{u}=\infty$. Тем самым, формула (44) дает пример стохастического интегрального представления для функционала от броуновского движения с бесконечным математическим ожиданием.

\section{СПИСОК ЛИТЕРАТУРЫ}

1. Clark I.M.C. The representation of functionals of Brownian motion by stochastic integrals. - Ann. Math. Statist., 1970, v. 41, № 4, p. 1282-1295.

2. Itô K. Multiple Wiener integral. - J. Math. Soc. Japan, 1951, v. 3, № 1, p. 157-169.

3. Revuz D., Yor M. Continuous Martingales and Brownian Motion. Berlin-Heidelberg: Springer-Verlag, 1994, $560 \mathrm{p}$.

4. Rogers L. C. G., Williams D. Diffusions, Markov Processes, and Martingales. V. 2: Itô Calculus. Chichester: Wiley, 1987, $475 \mathrm{p}$.

5. Липчер P. Ш., Ширяев A. Н. Статистика случайных процессов. М.: Наука, 1974.

6. Graversen S.E., Peskir G., Shiryaev A.N. Stopping Brownian motion without anticipation as close as possible to its ultimate maximum. - Теория вероятн. и еe примен., 2000 , т. 45 , в. 1 , с. $125-136$.

7. Ширяев А.Н. Основы стохастической финансовой математики. Т. 1: Факты, модели. Т. 2: Теория. М.: ФАЗИС, 1998, 512 с., 544 с.

8. Kunita H. Some extensions of Itô's formula. - Lecture Notes in Math., 1981, v. 850, p. 118-141.

9. Вентчель $A$. Д. Об уравнениях теории условных марковских процессов. - Теория вероятн. и ее примен., 1965 , т. 10, в. 2, с. 390-393. 